\title{
Unique Evolution of Symbiobacterium thermophilum Suggested from Gene Content and Orthologous Protein Sequence Comparisons
}

\author{
Kenro Oshima, ${ }^{1}$ Kenji Ueda, ${ }^{2}$ Teruhiko Beppu, ${ }^{2}$ and Hiromi Nishida ${ }^{3}$ \\ ${ }^{1}$ Department of Agricultural and Environmental Biology, Graduate School of Agricultural and Life Sciences, The University of Tokyo, \\ Bunkyo-ku, Tokyo 113-8657, Japan \\ ${ }^{2}$ Life Science Research Center, College of Bioresource Sciences, Nihon University, 1866 Kameino, Fujisawa 252-8510, Japan \\ ${ }^{3}$ Agricultural Bioinformatics Research Unit, Graduate School of Agriculture and Life Sciences, The University of Tokyo, Bunkyo-ku, \\ Tokyo 113-8657, Japan \\ Correspondence should be addressed to Hiromi Nishida, hnishida@iu.a.u-tokyo.ac.jp
}

Received 21 September 2010; Accepted 27 November 2010

Academic Editor: Hideaki Nojiri

Copyright (๑) 2011 Kenro Oshima et al. This is an open access article distributed under the Creative Commons Attribution License, which permits unrestricted use, distribution, and reproduction in any medium, provided the original work is properly cited.

Comparisons of gene content and orthologous protein sequence constitute a major strategy in whole-genome comparison studies. It is expected that horizontal gene transfer between phylogenetically distant organisms and lineage-specific gene loss have greater influence on gene content-based phylogenetic analysis than orthologous protein sequence-based phylogenetic analysis. To determine the evolution of the syntrophic bacterium Symbiobacterium thermophilum, we analyzed phylogenetic relationships among Clostridia on the basis of gene content and orthologous protein sequence comparisons. These comparisons revealed that these 2 phylogenetic relationships are topologically different. Our results suggest that each Clostridia has a species-specific gene content because frequent genetic exchanges or gene losses have occurred during evolution. Specifically, the phylogenetic positions of syntrophic Clostridia were different between these 2 phylogenetic analyses, suggesting that large diversity in the living environments may cause the observed species-specific gene content. S. thermophilum occupied the most distant position from the other syntrophic Clostridia in the gene content-based phylogenetic tree. We identified 32 genes (14 under relaxed selection and 18 under functional constraint) evolving under Symbiobacterium-specific selection on the basis of synonymous-to-nonsynonymous substitution ratios. Five of the 14 genes under relaxed selection are related to transcription. In contrast, none of the 18 genes under functional constraint is related to transcription.

\section{Introduction}

Symbiobacterium thermophilum is a phylogenetically unique bacterium that effectively grows only in coculture with a cognate Geobacillus sp. [1]. 16S rDNA-based phylogenetic analysis has shown that it is actually a Gram-positive bacterium [2]. Although S. thermophilum phylogenetically belongs to Clostridia (low GC-content bacterial group), the genome of $S$. thermophilum has a high GC content $(68.7 \%)$ [3]. Furthermore, 2 recent independent analyses concluded that Symbiobacterium affiliates with Clostridia (a class of Firmicutes): Ding et al. [4] carried out genome-context network analysis of 195 fully sequenced representative species, including $S$. thermophilum, and we analyzed the concatenated alignment of ribosomal protein sequences [5].

In a previous phylogenetic analysis that was based on ribosomal protein sequence comparisons [5], S. thermophilum was closely related to 6 recently sequenced Clostridia that have distinct properties, that is, Carboxydothermus hydrogenoformans, Desulfitobacterium hafniense, Moorella thermoacetica, Pelotomaculum thermopropionicum, Desulfotomaculum reducens, and Syntrophomonas wolfei. Symbiobacterium is dependent on the multiple functions of Geobacillus, including the supply of $\mathrm{CO}_{2}$ [1]. C. hydrogenoformans [6] grows by utilizing $\mathrm{CO}$ as a sole carbon source and water as an electron acceptor, which produces $\mathrm{CO}_{2}$ 
and hydrogen as waste products. D. hafniense [7] carries out anaerobic dechlorination of tetrachloroethene (PCE). M. thermoacetica [8] is an acetogenic bacterium that has been widely used to study the Wood-Ljungdahl pathway of $\mathrm{CO}$ and $\mathrm{CO}_{2}$ fixation (reductive acetyl-CoA pathway). $P$. thermopropionicum [9] is a member of a complex anaerobic microbial consortium where it catalyzes the intermediate bottleneck step by digesting volatile fatty acids (VFAs) and alcohols produced by upstream fermenting bacteria and it supplies acetate, hydrogen, and $\mathrm{CO}_{2}$ to downstream methanogenic archaea. D. reducens is an anaerobic sulfatereducing bacterium [10]. S. wolfei is a fatty-acid-degrading hydrogen/formate-producing anaerobic bacterium [11].

Comparisons of gene content and orthologous protein sequence constitute the major strategy in the whole-genome comparison study [12]. Clostridia have the large amount of bacteria. The phylogenetic position of Symbiobacterium remains uncertain in Clostridia. In this study, we reconstructed phylogenetic trees of Clostridia on the basis of the 2 different methods and compared them.

\section{Methods}

2.1. Phylogenetic Analysis on the Basis of Gene Content Comparisons. We used the following 51 bacteria (50 Clostridia and 1 Bacillus belonging to Firmicutes) in this analysis: Alkaliphilus metalliredigens, Alkaliphilus oremlandii, Ammonifex degensii, Anaerocellum thermophilum, Anaerococcus prevotii, Bacillus subtilis, Caldicellulosiruptor saccharolyticus, Candidatus Desulforudis audaxviator, Carboxydothermus hydrogenoformans, Clostridium acetobutylicum, Clostridium beijerinckii, Clostridium botulinum A ATCC 19397, C. botulinum A ATCC 3502, C. botulinum A Hall, C. botulinum A2, C. botulinum A3 Loch Maree, C. botulinum B Eklund 17B, C. botulinum B1 Okra, C. botulinum Ba4, C. botulinum E3, C. botulinum F Langeland, Clostridium cellulolyticum, Clostridium difficile 630, C. difficile CD196, Clostridium kluyveri DSM 555, C. kluyveri NBRC 12016, Clostridium novyi, Clostridium perfringens ATCC 13124, C. perfringens SM101, C. perfringens 13, Clostridium phytofermentans, Clostridium tetani E88, Clostridium thermocellum, Coprothermobacter proteolyticus, Desulfitobacterium hafniense DCB2, D. hafniense Y51, Desulfotomaculum acetoxidans, Desulfotomaculum reducens, Eubacterium eligens, Eubacterium rectale, Finegoldia magna, Halothermothrix orenii, Heliobacterium modesticaldum, Moorella thermoacetica, Natranaerobius thermophilus, Pelotomaculum thermopropionicum, Symbiobacterium thermophilum, Syntrophomonas wolfei, Thermoanaerobacter pseudethanolicus, Thermoanaerobacter sp. $\mathrm{X} 514$, and Thermoanaerobacter tengcongensis. Ortholog cluster analysis among the above 51 bacteria was performed using the MBGD [13] (Microbial Genome Database for Comparative Analysis; http://mbgd.nibb.ac.jp/). The analysis (minimum cluster size, 2) provided a gene presence/absence data matrix $(10,636$ genes $\times 51$ organisms $)$, which served as the basis for a distance matrix between all pairs of the 51 organisms. The distance was calculated from the different ratios between the presence/absence patterns of the 10,636 genes. On the basis of distance matrix, a neighbor-joining tree was reconstructed using MEGA software version 4 [14]. The bootstrap was performed with 1000 replicates.

2.2. Phylogenetic Analysis on the Basis of 112 Orthologous Protein Sequence Comparisons. We used the following 55 bacteria (54 Clostridia and 1 Bacillus) in this analysis: Acidaminococcus fermentans, A. metalliredigens, A. degensii, A. thermophilum, A. prevotii, B. subtilis, C. saccharolyticus, Candidatus D. audaxviator, C. hydrogenoformans, Clostridiales genomosp. BVAB3 UPII9-5, C. acetobutylicum, C. beijerinckii, C. botulinum A ATCC 19397, C. botulinum A ATCC 3502, C. botulinum A Hall, C. botulinum A2 Kyoto, C. botulinum A3 Loch Maree, C. botulinum B Eklund 17B, C. botulinum B1 Okra, C. botulinum Ba4 657, C. botulinum E3 Alaska E43, C. botulinum F Langeland, C. cellulolyticum, C. difficile 630, C. difficile CD196, C. difficile R20291, C. kluyveri DSM 555, C. kluyveri NBRC 12016, C. novyi, C. perfringens ATCC 13124, C. perfringens SM101, C. perfringens 13, C. phytofermentans, C. tetani, C. thermocellum, C. proteolyticus, D. hafniense DCB-2, D. hafniense Y51, D. acetoxidans, D. reducens, E. eligens, E. rectale, F. magna, $H$. orenii, $H$. modesticaldum, $M$. thermoacetica, $N$. thermophilus, $P$. thermopropionicum, S. thermophilum, S. wolfei, Thermoanaerobacter italicus, T. pseudethanolicus, T. sp. X514, T. tengcongensis, and Veillonella parvula. From the above 55 bacteria, 112 proteins were extracted as orthologous proteins by using a previously described method [15]. Thus, we constructed 112 multiple alignments using Clustal W [16]. Then, a concatenated multiple alignment of the 112 multiple alignments was generated. The complete multiple alignment had 52,204 amino acid sites, including 19,818 gap/insertion sites. Hence, phylogenetic analyses were performed on the basis of 32,386 amino acid sites without the gap/insertion sites. The neighbor-joining tree was reconstructed using MEGA software version 4 [14]. The bootstrap was performed with 1000 replicates. The rate variation among sites was considered to have a gamma-distributed rate $(\alpha=1)$. The other default parameters (e.g., Poisson distance) were not changed.

2.3. Extraction of Genes Evolving under SymbiobacteriumSpecific Selection among Syntrophic Clostridia. Among Bacillus subtilis, Carboxydothermus hydrogenoformans, Desulfitobacterium hafniense, Moorella thermoacetica, Pelotomaculum thermopropionicum, Desulfotomaculum reducens, Symbiobacterium thermophilum, and Syntrophomonas wolfei, 472 genes were extracted as orthologous genes by the previously described method [15]. Synonymous substitution occurs more frequently than nonsynonymous substitution in protein-coding sequences because of relaxed functional constraints (nonsynonymous-to-synonymous ratio $\omega<1$ ) [17], whereas they occur equally in noncoding regions and pseudogenes $(\omega=1)$. We calculated the likelihood of both the codon substitution model allowing for one $\omega$ (model R1) and the S. thermophilum branch-specific model allowing for 2 ratios ( $\omega_{0}$ and $\omega_{1}$; model R2), using PAML version 3.14 [18]. In model R2, the branches of the gene tree were partitioned into the Symbiobacterium branch $\left(\omega_{1}\right)$ and other related branches $\left(\omega_{0}\right)$. Likelihood ratio test statistics 
were calculated as twice the difference between the 2 loglikelihoods $(2 \Delta \ln )$ and compared with a $\chi^{2}$ distribution with degrees of freedom equal to the difference in the number of parameters between the 2 models [19]. According to this method, the genes evolving under the Symbiobacteriumspecific selection among Bacillus and 7 Clostridia were extracted.

\section{Results and Discussion}

Phylogenetic relationships among Clostridia on the basis of gene content comparison (Figure 1) were topologically different from those generated on the basis of orthologous protein sequence comparison (Figure 2). For example, in the gene content-based phylogenetic tree, Alkaliphilus, Clostridium (except for C. cellulolyticum and C. thermocellum), Desulfitobacterium, and Eubacterium formed a monophyletic lineage with $85 \%$ bootstrap support (Figure 1). In contrast, in the 112 orthologous protein sequence-based phylogenetic relationships, Alkaliphilus, Anaerococcus, Clostridium (except for C. cellulolyticum and C. thermocellum), Eubacterium, and Finegoldia formed a monophyletic lineage with $98 \%$ bootstrap support (Figure 2). Thus, the phylogenetic positions of Anaerococcus, Desulfitobacterium, and Finegoldia were different between these 2 trees. In addition, Coprothermobacter proteolyticus was positioned differently in the 2 trees. Moreover, the very long branch in the orthologous proteinbased tree suggests that $C$. proteolyticus has a substitution pattern that is different from other related Clostridia.

We expected horizontal gene transfer between phylogenetically distant organisms and lineage-specific gene loss to have greater influence on the gene content-based phylogenetic analysis than the orthologous protein-based analysis $[12,20]$. Bacteria make their gene content suitable for the living environment by changing it through gene acquisition and loss.

The phylogenetic positions of $2 D$. hafniense strains are located near those of Alkaliphilus, Clostridium (except for $C$. cellulolyticum and C. thermocellum), and Eubacterium in the gene content-based phylogenetic tree (Figure 1). However, those phylogenetic positions were located in the phylogenetic lineage of syntrophic Clostridia in the orthologous proteinbased tree (Figure 2). The gene content-based phylogenetic tree (Figure 1) indicates that Symbiobacterium branched off at the earliest stage of Clostridia species diversification. In contrast, Natranaerobius branched off at the earliest species diversification stage in the orthologous protein sequencebased phylogenetic tree (Figure 2).

Although S. thermophilum occupied the most basal position in the gene content-based Clostridia lineage (Figure 1), it was located in the syntrophic Clostridia lineage on the basis of orthologous protein sequence comparisons (Figure 2). Syntrophic bacteria evolved to acquire different sets of genes despite their close phylogenetic relationship. Thus, although Symbiobacterium clusters with syntrophic Clostridia, its gene content is very different. S. thermophilum has the most distant position from the other syntrophic Clostridia in the phylogenetic tree on the basis of gene content comparisons.
Although the physiological reason for the high $\mathrm{CO}_{2}$ requirement of $S$. thermophilum is not yet known, we assumed that it is related to the carbonic anhydrase deficiency (the ubiquitous enzyme catalyzing interconversion between $\mathrm{CO}_{2}$ and bicarbonate; EC 4.2.1.1), as deficiency of this enzyme results in the need for high $\mathrm{CO}_{2}$ levels in several model microorganisms [1]. S. thermophilum lost this enzyme in the course of evolution [5]. In this previous analysis, we inferred that $C$. hydrogenoformans and $M$. thermoacetica have also lost the gene for carbonic anhydrase; however, we recently noticed that $C$. hydrogenoformans had 2 potential carbonic anhydrase coding genes with structures different from the other syntrophic Clostridia carbonic anhydrases. Therefore, only Moorella has lost the carbonic anhydrase gene, in addition to Symbiobacterium. However, according to our results, these two bacteria are not closely related to each other (Figures 1 and 2), suggesting that the gene loss in these 2 species occurred independently during evolution.

Our results imply that each syntrophic Clostridial organism, especially Symbiobacterium, would have genes that evolved in an organism-specific manner. We expect that characterization of such genes will provide useful information with regard to the evolutionary history and physiological features specific to the corresponding organism [21, 22]. We identified 32 genes evolving under Symbiobacterium-specific selection (Table 1). The analysis revealed that the likelihood of model R2 was significantly higher $(P<.05)$ than that of model R1 in the 32 genes. Of these, 14 genes showed $\omega_{1} / \omega_{0}>1$ and 18 showed $\omega_{1} / \omega_{0}<1$.

Among the 32 genes evolving under Symbiobacteriumspecific selection, the RNA chaperone Hfq-coding gene has the highest $\omega_{1}$ value $(0.5347)$ (Table 1). Hfq facilitates pairing interactions between small regulatory RNAs and their mRNA targets, which has a variety of functions in bacteria [23]. Among 73 conserved amino acid sites of Hfq (Figure 3), S. thermophilum has more specific sites (7 sites) than the outgroup Bacillus (4 sites), indicating that the Hfq gene is one of the genes evolving under Symbiobacterium-specific selection.

Two genes related to transcription, sigA (RNA polymerase sigma factor coding gene) and rpoC (RNA polymerase subunit beta' coding gene) have evolved under relaxed selection (Table 1). These results could be related to the high GC content of Symbiobacterium genes. Thus, we hypothesized that the GC bias of the promoter sequence induced Symbiobacterium-specific SigA, a DNA-binding protein, which led to the structural change of RNA polymerase complex (including RpoC). We discussed the relationships between the GC content and phylogeny of the Symbiobacterium genes [24].

In addition, spoIIAB and cheY are also related to transcription. Thus, 5 of the 14 genes under more relaxed selection than other Clostridia are related to transcription. However, none of the 18 genes under functional constraint is related to transcription. Those results suggest that, under relaxed selection, the transcription system may be related to S. thermophilum-specific gene content. In fact, Symbiobacterium lost the transcriptional regulator genes arsR, GntR, and $\operatorname{Lrp}$ compared to other syntrophic Clostridia 


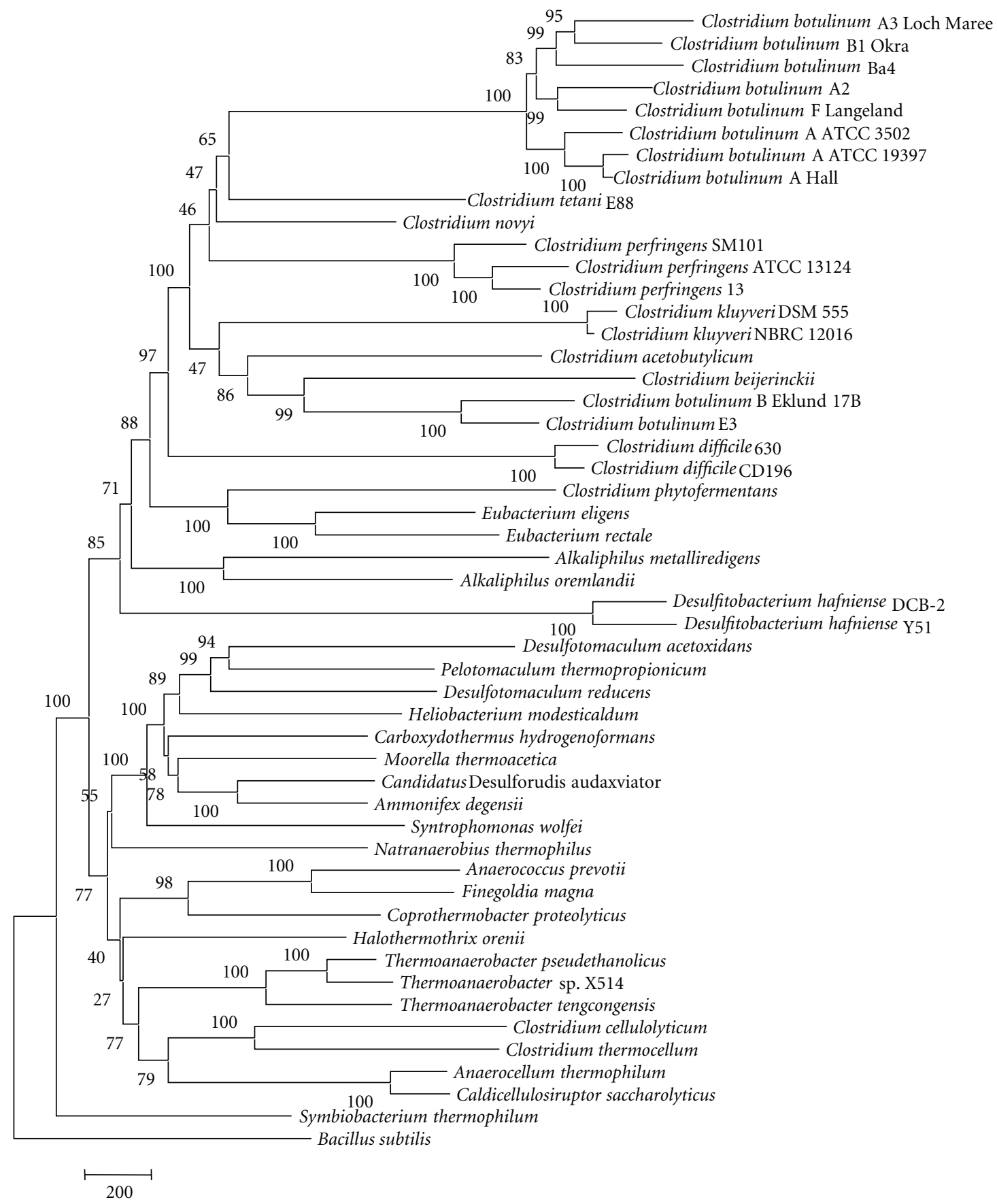

Figure 1: Phylogenetic relationships on the basis of gene content comparisons among 50 Clostridia and Bacillus subtilis. The ortholog cluster analysis (minimum cluster size, 2) among the 51 bacteria was performed using the MBGD [13]. This analysis produced the gene presence/absence data matrix (10,636 genes $\times 51$ organisms), which was used to generate the distance matrix between all pairs of the 51 bacteria. On the basis of the distance matrix, a neighbor-joining tree was reconstructed using MEGA software version 4 [14]. The bootstrap was performed with 1000 replicates. The bar indicates a 200-gene difference.

(See in the Supplementary Material available online at doi: $10.4061 / 2011 / 376831$ Table S1.).

It is noteworthy that some functionally related genes exhibited opposite nucleotide substitution patterns in $S$. thermophilum (Table 1). For example, $\operatorname{argD}(\mathrm{N}$ acetylornithine aminotransferase coding gene) has evolved under relaxed selection whereas $\operatorname{argC}(\mathrm{N}$-acetyl-gammaglutamyl-phosphate reductase coding gene) has evolved under functional constraint. Another example is the genes encoding flagella-associated proteins; flgG (flagellar hook protein coding gene) has evolved under relaxed selection, whereas flgD (flagellar hook assembly protein coding gene) 


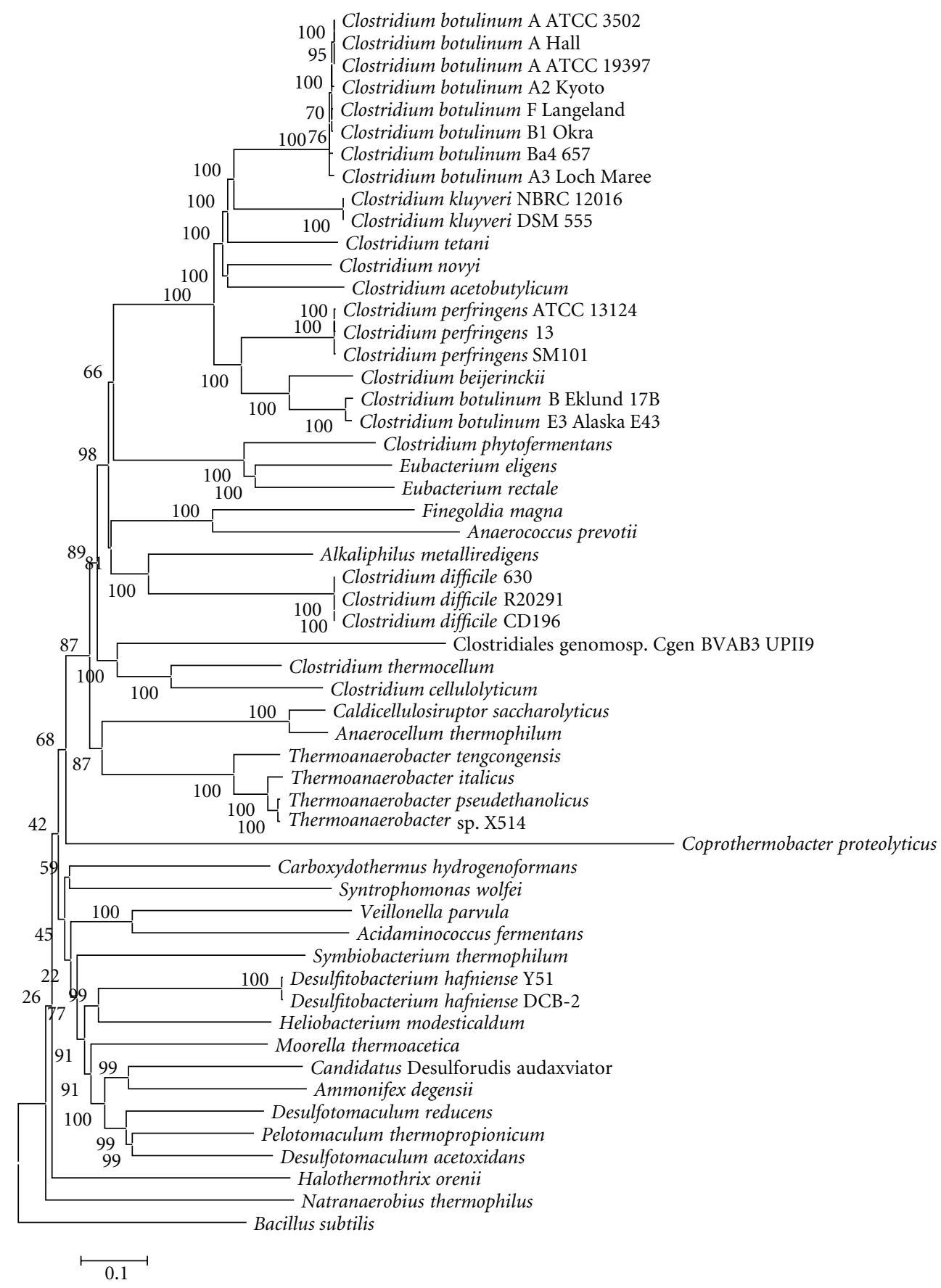

FIGURE 2: Phylogenetic relationships on the basis of 112 orthologous protein sequence comparisons among 54 Clostridia and B. subtilis. The 112 proteins were extracted as orthologous proteins from the 55 bacteria by a previously described method [15]. We constructed the 112 multiple alignments by using Clustal W [16]. Then, a concatenated multiple alignment of the 112 multiple alignments was generated. The complete multiple alignment had 52,204 amino acid sites, including 19,818 gap/insertion sites. Hence, phylogenetic analyses were performed on the basis of 32,386 amino acid sites without the gap/insertion sites. The neighbor-joining tree was reconstructed using MEGA software version 4 [14]. The bootstrap was performed with 1000 replicates. The rate variation among sites was assumed to have a gamma distributed rate $(\alpha=1)$. No other default parameters were changed. The bar indicates a $10 \%$ difference.

and fliS (flagellar protein coding gene) have evolved under functional constraint. flgG exhibited the highest $\omega_{1} / \omega_{0}$ value (75.48) (Table 1). Flagella mediate interactions between $P$. thermopropionicum and methanogenic archaea [25]. Similar specialized functions in syntrophic association could have been a limiting factor for the evolution of the above 2 flagellum genes in Symbiobacterium.

In conclusion, our results suggest that $S$. thermophilum has evolved in a unique manner compared to other syntrophic Clostridia from the perspective of gene content. 
TABle 1: Genes evolving under Symbiobacterium-specific selection.

\begin{tabular}{|c|c|c|c|}
\hline Gene & $\omega_{1}$ & $\omega_{1} / \omega_{0}$ & $2 \Delta \ln$ \\
\hline \multicolumn{4}{|l|}{$\omega_{1} / \omega_{0}>1$} \\
\hline hfq (RNA chaperone, STH1746) & 0.5347 & 24.3046 & 5.7413 \\
\hline spoIIAB (anti-sigma F factor, STH1813) & 0.3967 & 5.9744 & 8.7835 \\
\hline flgG (flagellar hook protein, STH2995) & 0.3774 & 75.4800 & 6.4323 \\
\hline ilvC (ketol-acid reductoisomerase, STH2688) & 0.2240 & 3.4675 & 10.7272 \\
\hline rplL (50S ribosomal protein L7/L12, STH3086) & 0.2183 & 8.3640 & 13.4750 \\
\hline $\begin{array}{l}\operatorname{argD}(N \text {-acetylornithine aminotransferase, } \\
\text { STH2881) }\end{array}$ & 0.2084 & 2.0292 & 4.1224 \\
\hline rplK (50S ribosomal protein L11, STH3090) & 0.1869 & 9.3450 & 4.2192 \\
\hline $\begin{array}{l}y \operatorname{lmE} \text { (alanine racemase domain-containing } \\
\text { protein, STH1227) }\end{array}$ & 0.1526 & 24.2222 & 15.4681 \\
\hline proJ (gamma-glutamyl kinase, STH2540) & 0.1497 & 26.2632 & 4.4715 \\
\hline sigA (RNA polymerase sigma factor, STH0588) & 0.1315 & 3.7679 & 17.9996 \\
\hline $\begin{array}{l}\text { rpoC (RNA polymerase subunit beta', } \\
\text { STH3084) }\end{array}$ & 0.0838 & 2.1487 & 7.2876 \\
\hline $\begin{array}{l}\text { glmS (glucosamine-fructose-6-phosphate } \\
\text { aminotransferase, STH1279) }\end{array}$ & 0.0156 & 2.7857 & 13.0700 \\
\hline $\begin{array}{l}\operatorname{aroE} \text { (3-phosphoshikimate } 1 \\
\text { carboxyvinyltransferase, STH1419) }\end{array}$ & 0.0125 & 2.8409 & 4.4748 \\
\hline $\begin{array}{l}\text { cheY (two-component response regulator } \\
\text { involved in modulation of flagellar, STH1540) }\end{array}$ & 0.0044 & 2.9333 & 6.7786 \\
\hline \multicolumn{4}{|l|}{$\omega_{1} / \omega_{0}<1$} \\
\hline $\begin{array}{l}\text { flgD (flagellar hook assembly protein, } \\
\text { STH2996) }\end{array}$ & 0.0123 & 0.0715 & 4.4609 \\
\hline fliS (flagellar protein FliS, STH2976) & 0.0073 & 0.0885 & 4.0842 \\
\hline $\begin{array}{l}\text { yloM (ribosomal RNA small subunit } \\
\text { methyltransferase B, STH1349) }\end{array}$ & 0.0045 & 0.0441 & 12.0081 \\
\hline ftsH (cell division protease, STH3198) & 0.0040 & 0.0655 & 11.9908 \\
\hline $\begin{array}{l}\text { spoVFB (dipicolinate synthase subunit B, } \\
\text { STH1546) }\end{array}$ & 0.0039 & 0.0591 & 6.5852 \\
\hline rplW (50S ribosomal protein L23, STH3073) & 0.0039 & 0.1429 & 3.9835 \\
\hline trmD (tRNA methyltransferase, STH1470) & 0.0038 & 0.0574 & 5.7865 \\
\hline $\begin{array}{l}\operatorname{argC}(N \text {-acetyl-gamma-glutamyl-phosphate } \\
\text { reductase, STH2892) }\end{array}$ & 0.0038 & 0.0721 & 4.1368 \\
\hline rpsC (30S ribosomal protein S3, STH3069) & 0.0037 & 0.1504 & 4.1064 \\
\hline $\begin{array}{l}\operatorname{prfA} \text { (peptide chain release factor RF-1, } \\
\text { STH0073) }\end{array}$ & 0.0035 & 0.0750 & 6.3618 \\
\hline ligA (NAD-dependent DNA ligase, STH2825) & 0.0034 & 0.0654 & 4.5717 \\
\hline $\begin{array}{l}\text { spo0J (ParB-like nuclease domain-containing } \\
\text { protein, STH3332) }\end{array}$ & 0.0034 & 0.0397 & 10.1363 \\
\hline $\begin{array}{l}\text { ftsE (cell division ATP-binding protein, } \\
\text { STH0139) }\end{array}$ & 0.0027 & 0.0407 & 5.6285 \\
\hline metG (methionyl-tRNA synthetase, STH3252) & 0.0027 & 0.0470 & 4.1885 \\
\hline rplC (50S ribosomal protein L3, STH3075) & 0.0023 & 0.0920 & 4.3304 \\
\hline rplB (50S ribosomal protein L2, STH3072) & 0.0020 & 0.0617 & 3.9836 \\
\hline rpsH (30S ribosomal protein S8, STH3061) & 0.0018 & 0.1047 & 4.5829 \\
\hline $\begin{array}{l}\text { infA (translation initiation factor IF-1, } \\
\text { STH3052) }\end{array}$ & 0.0004 & 0.0234 & 4.7842 \\
\hline
\end{tabular}




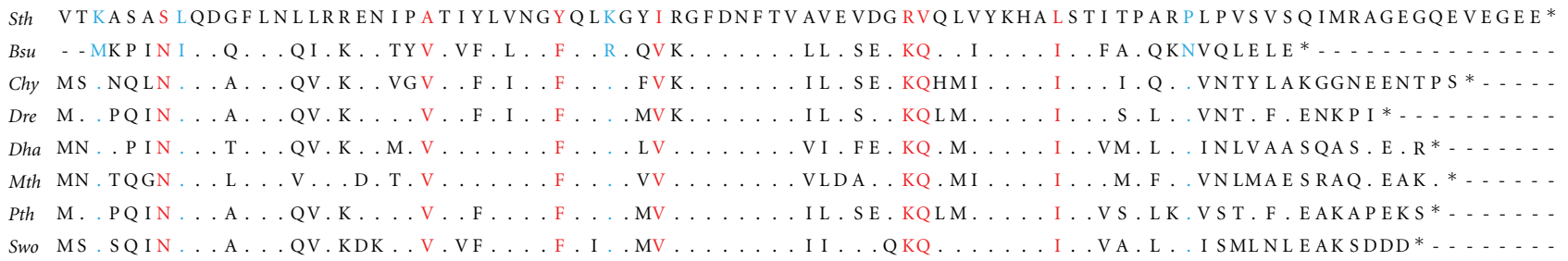

Figure 3: Alignment of amino acid sequences of Hfq. Sth, Symbiobacterium thermophilum; Bsu, Bacillus subtilis; Chy, Carboxydothermus hydrogenoformans; Dre, Desulfotomaculum reducens; Dha, Desulfitobacterium hafniense; Mth, Moorella thermoacetica; Pth, Pelotomaculum thermopropionicum; Swo, Syntrophomonas wolfei. Red and blue sites indicate Symbiobacterium- and Bacillus-specific sites, respectively. The dots represent identical residues of $S$. thermophilum amino acid.

Codon substitution analysis also suggests several unique genes that evolved in a Symbiobacterium-specific manner. Although speculative, the gene loss or relaxed evolution of several transcriptional regulator genes implies that environmental response might be involved in Symbiobacteriumspecific evolution.

\section{Acknowledgment}

This study was supported by the High-Tech Research Center Project of the Ministry of Education, Culture, Sports, Science and Technology, Japan.

\section{References}

[1] K. Ueda and T. Beppu, "Lessons from studies of Symbiobacterium thermophilum, a unique syntrophic bacterium," Bioscience, Biotechnology and Biochemistry, vol. 71, no. 5, pp. 1115-1121, 2007.

[2] M. Ohno, H. Shiratori, M. J. Park et al., "Symbiobacterium thermophilum gen. nov., sp. nov., a symbiotic thermophile that depends on co-culture with a Bacillus strain for growth," International Journal of Systematic and Evolutionary Microbiology, vol. 50, no. 5, pp. 1829-1832, 2000.

[3] K. Ueda, A. Yamashita, J. Ishikawa et al., "Genome sequence of Symbiobacterium thermophilum, an uncultivable bacterium that depends on microbial commensalism," Nucleic Acids Research, vol. 32, no. 16, pp. 4937-4944, 2004.

[4] G. Ding, Z. Yu, J. Zhao et al., "Tree of life based on genome context networks," PLoS One, vol. 3, no. 10, Article ID e3357, 2008.

[5] H. Nishida, T. Beppu, and K. Ueda, "Symbiobacterium lost carbonic anhydrase in the course of evolution," Journal of Molecular Evolution, vol. 68, no. 1, pp. 90-96, 2009.

[6] M. Wu, Q. Ren, A. S. Durkin et al., "Life in hot carbon monoxide: the complete genome sequence of Carboxydothermus hydrogenoformans Z-2901," PLoS Genetics, vol. 1, no. 5, p. e65, 2005.

[7] H. Nonaka, G. Keresztes, Y. Shinoda et al., "Complete genome sequence of the dehalorespiring bacterium Desulfitobacterium hafniense Y51 and comparison with Dehalococcoides ethenogenes 195," Journal of Bacteriology, vol. 188, no. 6, pp. 2262 2274, 2006.

[8] E. Pierce, G. Xie, R. D. Barabote et al., "The complete genome sequence of Moorella thermoacetica (f. Clostridium thermoaceticum)," Environmental Microbiology, vol. 10, no. 10, pp. 2550-2573, 2008.
[9] T. Kosaka, S. Kato, T. Shimoyama, S. Ishii, T. Abe, and K. Watanabe, "The genome of Pelotomaculum thermopropionicum reveals niche-associated evolution in anaerobic microbiota," Genome Research, vol. 18, no. 3, pp. 442-448, 2008.

[10] B. M. Tebo and A. Y. Obraztsova, "Sulfate-reducing bacterium grows with $\mathrm{Cr}(\mathrm{VI}), \mathrm{U}(\mathrm{VI}), \mathrm{Mn}(\mathrm{IV})$, and $\mathrm{Fe}(\mathrm{III})$ as electron acceptors," FEMS Microbiology Letters, vol. 162, no. 1, pp. 193198, 1998.

[11] M. J. McInerney, M. P. Bryant, R. B. Hespell, and J. W. Costerton, "Syntrophomonas wolfei gen. nov. sp. nov., an anaerobic, syntrophic, fatty acid-oxidizing bacterium," Applied and Environmental Microbiology, vol. 41, no. 4, pp. 1029-1039, 1981.

[12] Y. I. Wolf, I. B. Rogozin, N. V. Grishin, and E. V. Koonin, "Genome trees and the tree of life," Trends in Genetics, vol. 18, no. 9, pp. 472-479, 2002.

[13] I. Uchiyama, T. Higuchi, and M. Kawai, "MBGD update 2010: toward a comprehensive resource for exploring microbial genome diversity," Nucleic Acids Research, vol. 38, no. 1, pp. D361-D365, 2010.

[14] K. Tamura, J. Dudley, M. Nei, and S. Kumar, "MEGA4: molecular evolutionary genetics analysis (MEGA) software version 4.0," Molecular Biology and Evolution, vol. 24, no. 8, pp. 1596-1599, 2007.

[15] K. Oshima and H. Nishida, "Phylogenetic relationships among mycoplasmas based on the whole genomic information," Journal of Molecular Evolution, vol. 65, no. 3, pp. 249-258, 2007.

[16] J. D. Thompson, D. G. Higgins, and T. J. Gibson, "CLUSTAL $\mathrm{W}$ : improving the sensitivity of progressive multiple sequence alignment through sequence weighting, position-specific gap penalties and weight matrix choice," Nucleic Acids Research, vol. 22, no. 22, pp. 4673-4680, 1994.

[17] W. H. Li, T. Gojobori, and M. Nei, "Pseudogenes as a paradigm of neutral evolution," Nature, vol. 292, no. 5820, pp. 237-239, 1981.

[18] Z. Yang, "PAML: a program package for phylogenetic analysis by maximum likelihood," Computer Applications in the Biosciences, vol. 13, no. 5, pp. 555-556, 1997.

[19] N. Goldman and Z. Yang, "A codon-based model of nucleotide substitution for protein-coding DNA sequences," Molecular Biology and Evolution, vol. 11, no. 5, pp. 725-736, 1994.

[20] B. Snel, M. A. Huynen, and B. E. Dutilh, "Genome trees and the nature of genome evolution," Annual Review of Microbiology, vol. 59, pp. 191-209, 2005.

[21] K. Oshima and H. Nishida, "Detection of the genes evolving under Ureaplasma-specific selection," Journal of Molecular Evolution, vol. 66, no. 5, pp. 529-532, 2008. 
[22] H. Nishida, "Ureaplasma urease genes have undergone a unique evolutionary process," Open Systems Biology Journal, vol. 2, pp. 1-7, 2009.

[23] A. Jousselin, L. Metzinger, and B. Felden, "On the facultative requirement of the bacterial RNA chaperone, Hfq," Trends in Microbiology, vol. 17, no. 9, pp. 399-405, 2009.

[24] H. Nishida and C.-S. Yun, "Phylogenetic and guanine-cytosine content analysis of Symbiobacterium thermophilum genes," International Journal of Evolutionary Biology, vol. 2011, p. 5, 2011.

[25] T. Shimoyama, S. Kato, S. Ishii, and K. Watanabe, "Flagellum mediates symbiosis," Science, vol. 323, no. 5921, p. 1574, 2009. 

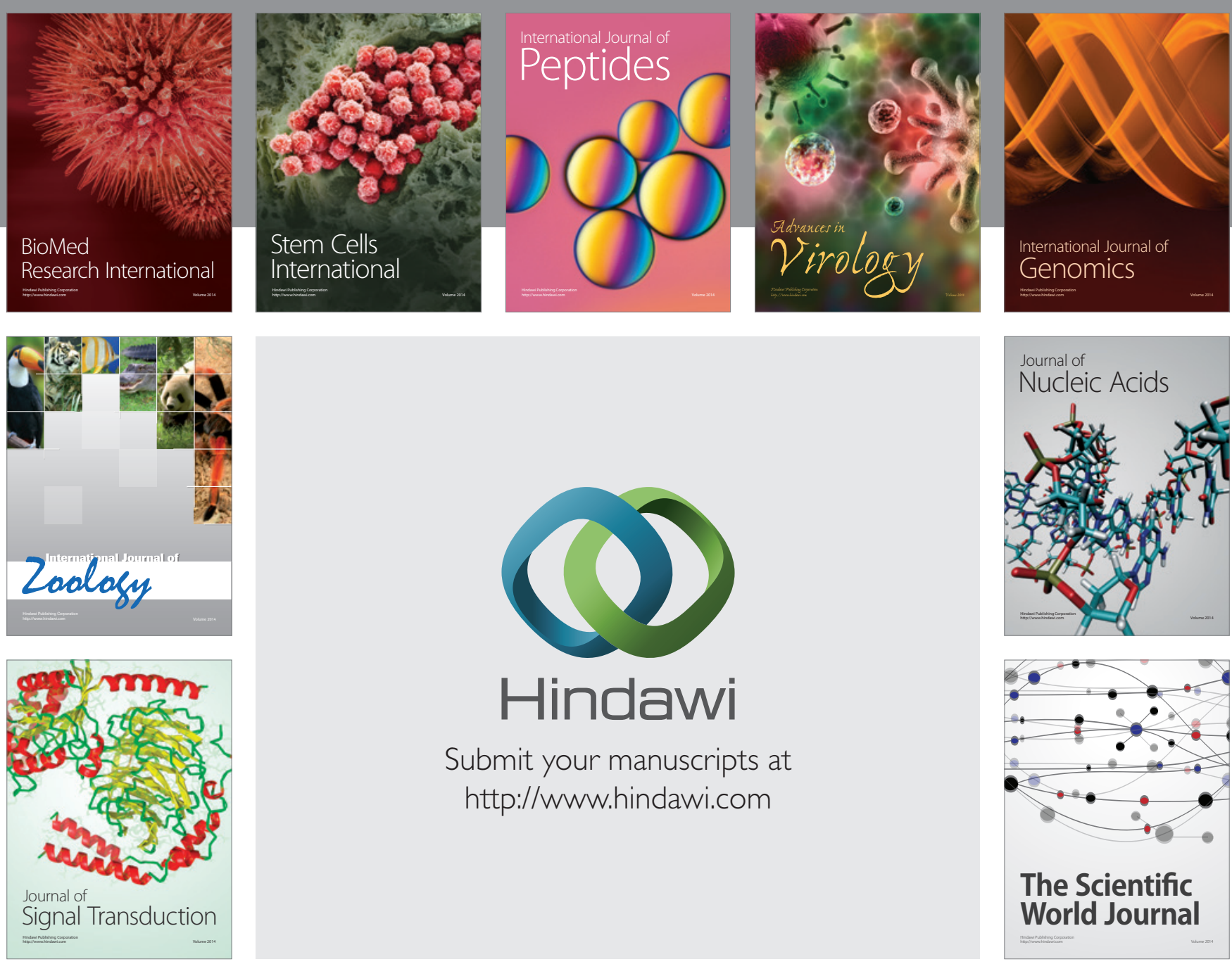

Submit your manuscripts at

http://www.hindawi.com
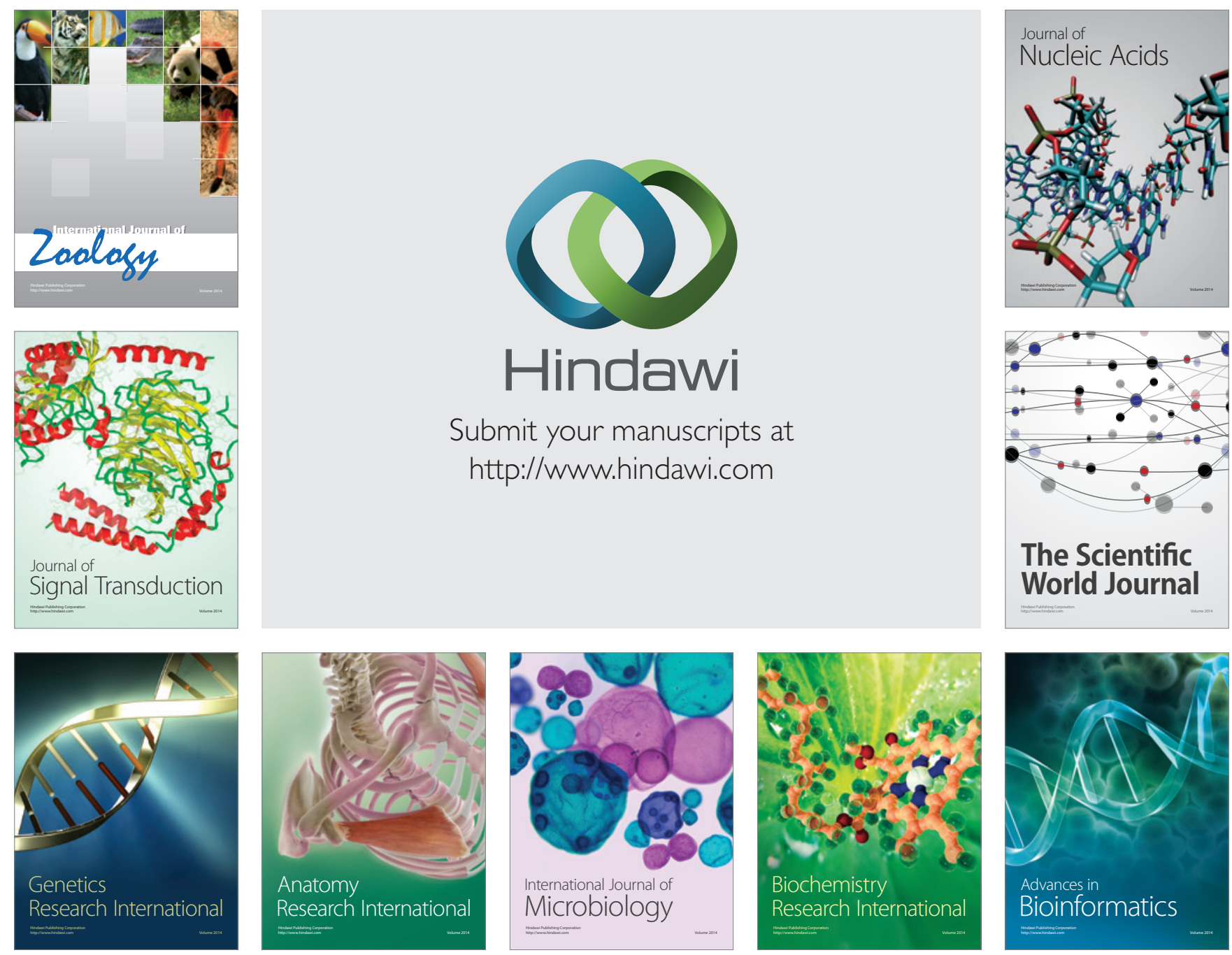

The Scientific World Journal
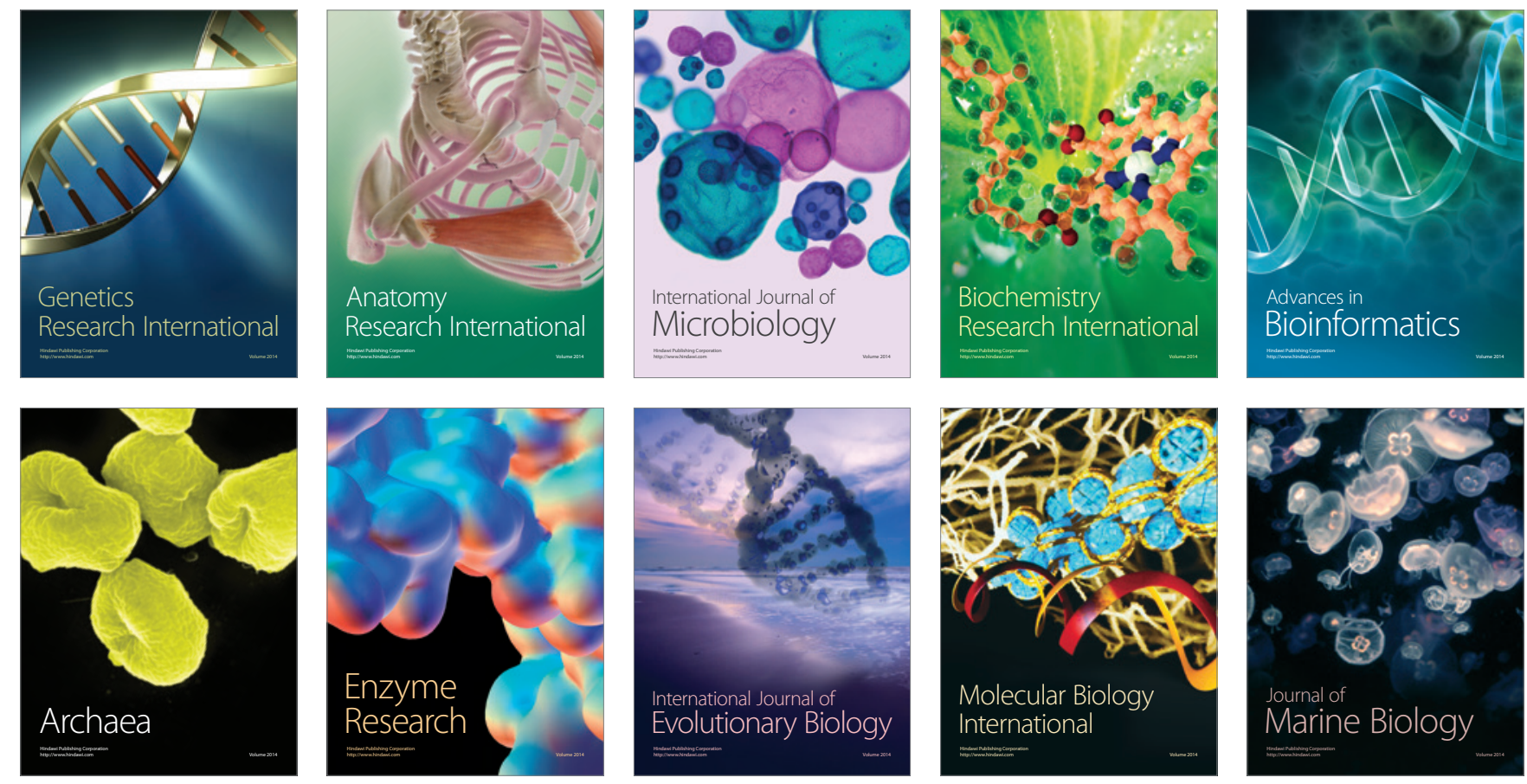